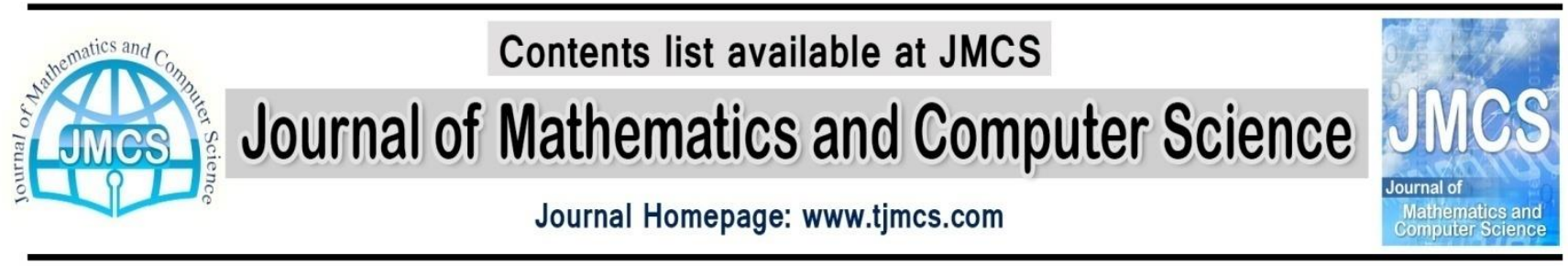

\title{
RECONSTRUCTION OF THE STURM-LIOUVILLE OPERATORS WITH TRANSMISSION AND PARAMETER DEPENDENT BOUNDARY CONDITIONS
}

\author{
Mostafa Fallahi, Fereshte Sharaghi, Mohammad Shahriari \\ Department of Mathematics, Faculty of Science, University of Maragheh, P.O. Box 55181-83111, \\ Maragheh, Iran.
}

fallahil_mostafa@yahoo.com,shareghi@deylaman.ac.ir,shahriari@tabrizu.ac.ir

Article history:

Received June 2014

Accepted July 2014

Available online September 2014

\section{Abstract}

Inverse problems of recovering the coefficients of discontinuous Sturm-Liouville problems with the eigenvalue parameter linearly contained in one of the boundary conditions are studied:

1) From Weyl m- function.

2) From spectral data.

Keywords: Inverse Sturm-Liouville problem, Weyl m-Function, discontinuous and parameter dependent boundary conditions.

\section{Introduction}

We consider the Sturm-Liouville problem

$$
l y=-y^{\prime \prime}+q y=\lambda y,
$$

With the eigenparameter dependent boundary conditions:

$$
\begin{aligned}
& U(y):=y^{\prime}(0)-h y(0)=0, \\
& V(y):=\left(\lambda-H_{1}\right) y^{\prime}(\pi)+\left(\lambda H-H_{2}\right) y(\pi)=0,
\end{aligned}
$$

And discontinuous conditions

$$
y(a+0)=\alpha_{1} y(a-0), y^{\prime}(a+0)=\alpha_{1}^{-1} y^{\prime}(a-0)+\alpha_{2} y(a-0),
$$

Where $\mathrm{q}(\mathrm{x}) \in \mathrm{L}_{2}(0, \pi)$ is a real-valued function, $\mathrm{h}, \mathrm{H}, \mathrm{H}_{1}, \mathrm{H}_{2}, \mathrm{a}, \alpha_{1}, \alpha_{2} \in \mathbb{R}$ and $\mathrm{r}=\mathrm{HH}_{1}-\mathrm{H}_{2}>$ 0 . For simplicity we use the notation $L=L\left(q(x), a, \alpha_{1}, \alpha_{2}, h, H, H_{1}, H_{2}\right)$ for the problem (1.1) (1.4) Boundary value problems with discontinuities inside the interval often appear in Mathematics, mechanics, physics, geophysics and other branches of natural sciences. As a rule, such 
problems are connected with discontinuous material properties. The inverse problem of reconstructing the material properties of a medium from data collected outside of the medium is of central importance in disciplines ranging from engineering to the geosciences. The inverse problem of recovering higher-order differential operators from the Weyl functions has been studied in [17]. In [1] the Sturm-Liouville problem with discontinuities in the case when an eigenparameter linearly appears not only in the differential equation but it also appears in both of the boundary conditions is investigated. Paper [14] is devoted to the study of inverse problems by (i) one spectrum and a sequence of norming constants; (ii) two spectra. We will first start in section 2 to obtain the spectral properties of $L$ and study the asymptotic behavior of eigenvalues, eigenfunction and norming constants with discontinuity in an interior point on $(0, \pi)$. In section 3 we study the inverse problem of recovering the pair $L=L\left(q(x), a, \alpha_{1}, \alpha_{2}, h, H, H_{1}, H_{2}\right)$ of the from (1.1) - (1.4) from the given Weyl function $\mathrm{M}(\lambda)$. For this purpose we will use the method of spectral mappings for SturmLiouville operators on interval $(0, a) \cup(a, \pi)$ and using the solution of the main equation, we provide algorithm 3.1 for the solution of the inverse problem. In section 4 we construction Sturm-Liouville equation with spectra data $\left\{\lambda_{n}, \gamma_{n}\right\}_{n \geq 0}$ by algorithm (4.1).

We refer to the somewhat complementary surveys in $[1,14,3,4,8,9,11,14,15,18]$ and $[20]$ for further aspects of this field. For general background on inverse Sturm-Liouville problems we refer (e.g.) to the monographs $[7,10,12,17,19]$ and [21].

\section{Properties of the spectrum}

Let $\varphi(\mathrm{x}, \lambda)$ and $\psi(x, \lambda)$ be the solutions of (1.1) satisfying the initial conditions

$$
\begin{array}{ll}
\varphi(0, \lambda)=1, & \varphi^{\prime}(0, \lambda)=h, \\
\psi(\pi, \lambda)=\lambda-H_{1}, & \psi^{\prime}(\pi, \lambda)=-\lambda H+H_{2}
\end{array}
$$

From the linear differential equations we obtain the Wronskian

$$
W(u, v):=u(x) v^{\prime}(x)-u^{\prime}(x) v(x)
$$

Is constant on $\mathrm{x} \in(0, \mathrm{a}) \cup(\mathrm{a}, \pi)$ for two solutions $l u=\lambda u, l v=\lambda v$ satisfying the transmission conditions (1.4). Moreover, we set

$$
\chi(\lambda):=W(\varphi(\lambda), \psi(\lambda))=V(\varphi)=-U(\psi) .
$$

Then $\chi(\lambda)$ is an entire function whose roots $\lambda_{n}$ coincide with the eigenvalues of $L$. Let the inner product in the Hilbert space $\mathrm{H}=\mathrm{L}_{2}(0, \pi) \oplus \mathrm{C}$ be define by

$$
\langle F, G\rangle_{\mathbb{H}}=\int_{0}^{\pi} \mathrm{F}_{1}(\mathrm{x}) \overline{\mathrm{G}}_{1}(\mathrm{x}) \mathrm{dx}+\frac{1}{\mathrm{r}} \mathrm{F}_{2} \overline{\mathrm{G}}_{2},
$$

Where

$$
\mathrm{F}=\left(\begin{array}{c}
\mathrm{F}_{1}(\mathrm{x}) \\
\mathrm{F}_{2}
\end{array}\right) \text { and } \mathrm{G}=\left(\begin{array}{c}
\mathrm{G}_{1}(\mathrm{x}) \\
\mathrm{G}_{2}
\end{array}\right) \in \mathbb{H} \text {. }
$$

We define the operator T acting in $\mathbb{H}$ such that

$$
\mathrm{T}(\mathrm{F})=\left(\begin{array}{l}
-\mathrm{F}^{\prime \prime}(\mathrm{x})+\mathrm{q}(\mathrm{x}) \mathrm{F}_{1}(\mathrm{x}) \\
\mathrm{H}_{1} \mathrm{~F}_{1}^{\prime}(\pi)+\mathrm{H}_{2} \mathrm{~F}_{1}(\pi)
\end{array}\right)
$$

With 


$$
D(T)=\left\{\begin{array}{l|l}
F \in \mathbb{H} & \begin{array}{c}
F_{1}(x), F_{1}^{\prime}(x) \in A C[0, a) \cup(a, \pi], l F_{1} \in L_{2}(0, \pi), \\
F_{1}^{\prime}(a+0)=\alpha_{1}^{-1} F_{1}^{\prime}(a-0)+\alpha_{2} F_{1}(a-0), \\
F_{1}(a+0)=\alpha_{1} F_{1}(a-0), F_{2}=F_{1}^{\prime}(\pi)+H F_{1}(\pi)
\end{array}
\end{array}\right\}
$$

Denote

$$
\Phi_{n}(x)=\left(\begin{array}{c}
\varphi\left(x, \lambda_{n}\right) \\
\varphi^{\prime}\left(\pi, \lambda_{n}\right)+H \varphi\left(\pi, \lambda_{n}\right)
\end{array}\right)
$$

It is easy to see that the set of functions $\left\{\Phi_{n}\right\}, n \geq 0$ are orthogonal function, i.e.

$$
\left\langle\Phi_{\mathrm{n}}, \Phi_{\mathrm{m}}\right\rangle=0, \quad n \neq m \text {. }
$$

By attaching a subscript 1 or 2 to the functions $\varphi$ and $\psi$, we mean to refer to the first subinterval [0, a) or to the second subinterval $(a, \pi]$. Therefore we see that

$$
\varphi\left(x, \lambda_{n}\right)= \begin{cases}\varphi_{1}\left(x, \lambda_{n}\right), & x<a \\ \varphi_{2}\left(x, \lambda_{n}\right), & x>a\end{cases}
$$

Therefore, we define norming constants by

$$
\gamma_{n}=\left\|\Phi_{n}\right\|^{2}=\int_{0}^{a} \varphi_{1}^{2}\left(x, \lambda_{n}\right) d x+\int_{a}^{\pi} \varphi_{2}^{2}\left(x, \lambda_{n}\right) d x+\frac{\left(\varphi_{2}^{\prime}\left(\pi, \lambda_{n}\right)+H \varphi_{2}\left(\pi, \lambda_{n}\right)\right)^{2}}{r}
$$

Where $\varphi_{1}\left(x, \lambda_{n}\right)$ and $\varphi_{2}\left(x, \lambda_{n}\right)$ are defined in Theorem 2.2.

Remark 2.1 The numbers $\left\{\lambda_{n}, \gamma_{n}\right\}_{n \geq 0}$ are called the spectral data of the problem (1.1)-(1.4).

Theorem 2.2 The following asymptotic forms hold

$$
\begin{gathered}
\sqrt{\lambda_{n}}=\rho_{n}=\rho_{n-3}^{0}+\frac{\delta_{n}}{n}+\frac{\xi_{n}}{n} \\
\varphi\left(x, \rho_{n}\right)= \begin{cases}-\left(\rho_{n-3}^{0}\right)^{2} \cos \rho_{n-3}^{0} x+O\left(\frac{1}{n}\right) & x<a \\
\left(\rho_{n-3}^{0}\right)^{2}\left[-\alpha^{+} \cos \rho_{n-3}^{0} x+\alpha^{-} \cos \rho_{n-3}^{0}(2 a-x)\right]+O\left(\frac{1}{n}\right) & x>a\end{cases} \\
\gamma_{n}=\left(\rho_{n-3}^{0}\right)^{4}\left[\frac{\pi-a}{2}\left(\left(\alpha^{+}\right)^{2}+\left(\alpha^{-}\right)^{2}\right)+\frac{a}{2}\right]+O\left(n^{3}\right)
\end{gathered}
$$

Where $\tau=\operatorname{Im} \rho, \alpha^{ \pm}=\frac{1}{2}\left(\alpha_{1} \pm \frac{1}{\alpha_{1}}\right), \xi_{n}=O(1), \delta_{n} \in l_{\infty}, \rho_{n}^{0}=n+h_{n}$, and $h_{n} \in l_{\infty}$.

Proof By using the similar proof of [17] we obtain

$$
\begin{aligned}
& \varphi(x, \rho)=\cos \rho x+\frac{h}{\rho} \sin \rho x+\frac{1}{\rho} \int_{0}^{x} \sin \rho(x-t) q(t) \varphi(t, \rho) d t \quad x<a, \\
& \varphi(x, \rho)=\alpha^{+} \cos \rho x+\alpha^{-} \cos \rho(2 a-x)+\left(h \alpha^{+}+\frac{\alpha_{2}}{2}\right) \frac{\sin \rho x}{\rho} \\
& +\left(h \alpha^{-}-\frac{\alpha_{2}}{2}\right) \frac{\sin \rho(2 a-x)}{\rho}+\frac{1}{\rho} \int_{a}^{x} \sin \rho(x-t) q(t) \varphi(t, \rho) d t \quad x>a,
\end{aligned}
$$


(2.17) $\quad \psi(x, \rho)=\left(H_{1}-\rho_{1}\right)\left(\alpha^{+} \cos \rho(\pi-x)-\alpha^{-} \cos \rho(x+\pi-2 a)\right)+$

$$
\begin{gathered}
+\left(\left(H_{2}-\rho^{2} H\right) \alpha^{+}+\frac{\alpha_{2}}{2}\right) \frac{\sin \rho(\pi-x)}{\rho}+\left(\left(H_{2}-\rho^{2} H\right) \alpha^{-}-\frac{\alpha_{2}}{2}\right) \frac{\sin \rho(x+\pi-2 a)}{\rho}+ \\
+\frac{1}{\rho} \int_{a}^{\pi}\left(\alpha^{+} \sin \rho(x-t)+\alpha^{-} \sin \rho(x+t-2 a)\right) q(t) \psi(t, \rho) d t \\
+\frac{1}{\rho} \int_{x}^{a} \sin \rho(t-x) q(t) \psi(t, \rho) d t \\
\end{gathered}
$$

(2.18) $\quad \psi(x, \rho)=\frac{H_{2}-\rho^{2} H_{0}}{\rho} \sin \rho(\pi-x)+\left(H_{1}-\rho^{2}\right) \cos \rho(\pi-x)$

$$
+\frac{1}{\rho} \int_{x}^{\pi} \sin \rho(t-x) q(t) \psi(t, \rho) d t \quad x>a .
$$

From $(2.15)-(2.18)$ we obtain

$$
\begin{aligned}
& \frac{\varphi(x, \rho)}{\rho^{2}}= \begin{cases}-\cos \rho x+O\left(\frac{1}{|\rho|} \exp |\tau| x\right) & x<a, \\
\left(-\alpha^{+} \cos \rho x+\alpha^{-} \cos \rho(2 a-x)\right)+O\left(\frac{1}{|\rho|} \exp |\tau| x\right) & x>a\end{cases} \\
& \frac{\psi(x, \rho)}{\rho^{2}}= \begin{cases}-\alpha^{+} \cos \rho(\pi-x)-\alpha^{-} \cos \rho(x+\pi-2 a)+O\left(\frac{1}{|\rho|} \exp |\tau|(\pi-x)\right), & x<a, \\
-\cos \rho(\pi-x)+O\left(\frac{1}{|\rho|} \exp |\tau|(\pi-x)\right) & x>a .\end{cases}
\end{aligned}
$$

From (2.4) and (2.19) - (2.20) we get

$$
\chi(\lambda)=\lambda^{3}\left(\alpha^{+} \frac{\sin \sqrt{\lambda} \pi}{\sqrt{\lambda}}+\alpha^{-} \frac{\sin \sqrt{\lambda}(2 a-\pi)}{\sqrt{\lambda}}\right)+O\left(\lambda^{2} \exp |\tau| \pi\right)
$$

\section{Denote}

$$
\chi_{0}(\lambda)=\alpha^{+} \frac{\sin \sqrt{\lambda} \pi}{\sqrt{\lambda}}+\alpha^{-\frac{\sin \sqrt{\lambda}(2 a-\pi)}{\sqrt{\lambda}}}
$$

And

$$
G_{n}=\left\{\lambda \in \mathbb{C} ; \lambda=\rho^{2},|\rho|=\left|\rho_{n}^{0}-\delta\right|\right\},
$$


Where $\delta$ is sufficiently small and $\rho_{\mathrm{n}}^{0}$ are the zeros of $\chi_{0}(\lambda)$ except 0 . Since, $\left|\chi_{0}(\lambda)\right| \geq C\left(\lambda^{\frac{5}{2}} \exp |\tau| \pi\right)$ And $\left|\chi(\lambda)-\chi_{0}(\lambda)\right|=O\left(\lambda^{2} \exp |\tau| \pi\right)$ for $\lambda \in G_{n}$ and large values $\mathrm{n}$, using the Rouche's theorem, We establish that contour $G_{n}$. Consequently, in the annulus between $G_{n}$ and $G_{n+1}, \chi$ has precisely one zero, namely $\rho_{n}^{2}$. Therefore, for the eigenvalue $\lambda_{n}$, the equality $\lambda_{n+3}=\rho_{n}^{2}$ is true. On the other hand, by using again the Rouche's theorem in $\gamma_{\varepsilon}=\left\{\lambda:\left|\lambda-\rho_{n}^{0}\right|<\varepsilon\right\}$ for sufficiently small $\varepsilon$, we get the asymptotic formulae $\rho_{n}=\rho_{n}^{0}+\varepsilon_{n}\left(\varepsilon_{n}=o(1)\right)$ is valid for large $n$. Finally, the equality $\varepsilon_{n}=O\left(\frac{1}{n}\right)$ is taken from the well known formulae $\chi_{0}(\lambda)\left(\rho_{n}^{0}+\varepsilon_{n}\right)=\chi_{0}^{\prime}\left(\rho_{n}^{0}\right) \varepsilon_{n}+o\left(\varepsilon_{n}\right)$, This fact proves the equality (2.12) where $\delta_{n}$ is the same as $\theta_{n}$ in ([6],p.240). By using (2.12) in (2.19) and (2.13) in (2.11) we get (2.13) and (2.14) respectively.

\section{Reconstruction by Weyl M-function}

Using properties of the spectrum the Weyl m-function [13], we can write

$$
m(\lambda)=-\frac{\psi(0, \lambda)}{\chi(\lambda)}
$$

Also asymptotic expansion have been obtained

$$
m(\lambda)=\frac{1}{\sqrt{-\lambda}}+O\left(\lambda^{-1}\right)
$$

Let $S(x, \lambda)$ be a solution of (1.1) subject to the initial conditions

$$
\mathrm{S}(0, \lambda)=0, \quad \mathrm{~S}^{\prime}(0, \lambda)=1
$$

and the jump conditions (1.4). The function $\psi(x, \lambda)$ can be represented as

$$
\theta(x, \lambda):=\frac{\psi(x, \lambda)}{\chi(\lambda)}=S(x, \lambda)-m(\lambda) \varphi(x, \lambda) .
$$

Where the functions $\theta(x, \lambda)$ and $m(\lambda)$ are called the Weyl solution and the Weyl function for the boundary value problem $L$. Now, we prove the uniqueness theorem for the solution of the inverse problem. We agree together a boundary value problem $\widetilde{L}$ of the same form but with different coefficients $\tilde{q}(x), \tilde{a}, \tilde{\alpha}_{1}, \tilde{\alpha}_{2}, \tilde{h}, \widetilde{H}, \widetilde{H}_{1}, \widetilde{H}_{2}$.

Theorem 3.1 If $M(\lambda)=\widetilde{M}(\lambda)$, then $L=\tilde{L}$, i.e, $q(x)=\tilde{q}(x)$, a.e, $a=\tilde{a}, \alpha_{1}=\tilde{\alpha}_{1}, \alpha_{2}=\tilde{\alpha}_{2}$

$$
, h=\tilde{h}, H=\widetilde{H}, H_{1}=\widetilde{H}_{1}, H_{2}=\widetilde{H}_{2} \text {. }
$$

Proof. Let us define the matrix $P(x, \lambda)=\left[P_{j k}(x, \lambda)\right], j ; k=1,2$ by the formula

$$
P(x, \lambda)\left(\begin{array}{cc}
\tilde{\varphi}(x, \lambda) & \widetilde{\Phi}(x, \lambda) \\
\tilde{\varphi}^{\prime}(x, \lambda) & \widetilde{\Phi}^{\prime}(x, \lambda)
\end{array}\right)=\left(\begin{array}{cc}
\varphi(x, \lambda) & \Phi(x, \lambda) \\
\varphi^{\prime}(x, \lambda) & \Phi^{\prime}(x, \lambda)
\end{array}\right)
$$

Using (3.2) and (3.4) we have,

$$
\left\{\begin{array}{l}
\varphi(x, \lambda)=P_{11}(x, \lambda) \tilde{\varphi}(x, \lambda)+P_{12}(x, \lambda) \tilde{\varphi}^{\prime}(x, \lambda), \\
\Phi(x, \lambda)=P_{11}(x, \lambda) \widetilde{\Phi}(x, \lambda)+P_{12}(x, \lambda) \widetilde{\Phi}^{\prime}(x, \lambda),
\end{array}\right.
$$

According to (3.1) and (3.5), for fixed $x$, the functions $P_{j k}(x, \lambda)$ are meromorphic Function in $\lambda$ with simple poles in the points $\lambda_{n}$ and $\tilde{\lambda}_{n}$. Denote $G_{\delta}^{0}=G_{\delta} \cap \tilde{G}_{\delta}$ where

$$
G_{\delta}=\left\{\lambda:\left|\lambda-\lambda_{n}\right|>\delta, n=1,2, \ldots\right\}
$$

And 


$$
\tilde{G}_{\delta}=\left\{\lambda:\left|\lambda-\tilde{\lambda}_{n}\right|>\delta, n=1,2, \ldots\right\}
$$

From $\rho \in G_{\delta}$ and using the asymptotic form of $\Phi^{v}(x, \lambda)$ we get $\left|\Phi^{v}(x, \lambda)\right| \leq C_{\delta}|\rho|^{v-3} \exp (-|\tau| x)$. Thus it follows that

$$
\left|P_{11}(x, \lambda)\right| \leq C_{\delta},\left|P_{12}(x, \lambda)\right| \leq C_{\delta}|\rho|^{-1}, \rho \in G_{\delta}^{0} .
$$

Using (3.1) and (3.5) we get

$$
\begin{aligned}
& P_{11}(x, \lambda)=\varphi(x, \lambda) \tilde{S}^{\prime}(x, \lambda)-S(x, \lambda) \tilde{\varphi}^{\prime}(x, \lambda)+(\tilde{M}(\lambda)-M(\lambda)) \varphi(x, \lambda) \tilde{\varphi}^{\prime}(x, \lambda) \\
& P_{12}(x, \lambda)=S(x, \lambda) \tilde{\varphi}(x, \lambda)-\varphi(x, \lambda) \tilde{S}(x, \lambda)+(\widetilde{M}(\lambda)-M(\lambda)) \varphi(x, \lambda) \tilde{\varphi}(x, \lambda) .
\end{aligned}
$$

Thus, if $M(\lambda) \equiv \widetilde{M}(\lambda)$, then for each fixed $x$, the function $P_{1 k}(x, \lambda)$ are entire in

$\lambda$. Together with (3.7) this yields $P_{12}(x, \lambda) \equiv 0, P_{11}(x, \lambda) \equiv A(x)$. Using (3.6) we

Derive

$$
\varphi(x, \lambda) \equiv A(x) \tilde{\varphi}(x, \lambda), \quad \Phi(x, \lambda) \equiv A(x) \widetilde{\Phi}(x, \lambda) .
$$

From $W(\Phi(x, \lambda), \varphi(x, \lambda)) \equiv 1$ and similarly $W(\widetilde{\Phi}(x, \lambda), \tilde{\varphi}(x, \lambda)) \equiv 1$, we have $A(x)=1$. So from (3.8) we obtain, $\varphi(x, \lambda) \equiv \tilde{\varphi}(x, \lambda)$ and $\Phi(x, \lambda) \equiv \widetilde{\Phi}(x, \lambda)$ for all $x$ and $\lambda$. Consequently, $L=$ $\tilde{L}$.

Now we construct the solution of the inverse problem. For this work first we denote

$$
\begin{cases}D(x, \lambda, \mu):=\frac{W(\varphi(x, \lambda), \varphi(x, \mu))}{\lambda-\mu}=\int_{0}^{x} \varphi(t, \lambda) \varphi(t, \mu) d t, & r(x, \lambda, \mu)=D(x, \lambda, \mu) \widehat{M}(\mu), \\ \widetilde{D}(x, \lambda, \mu):=\frac{W(\widetilde{\varphi}(x, \lambda), \widetilde{\varphi}(x, \mu))}{\lambda-\mu}=\int_{0}^{x} \tilde{\varphi}(t, \lambda) \tilde{\varphi}(t, \mu) d t, & \widetilde{r}(x, \lambda, \mu)=\widetilde{D}(x, \lambda, \mu) \widehat{M}(\mu),\end{cases}
$$

From Theorem 2.2 we have

$$
D(x, \lambda, \mu)= \begin{cases}\int_{0}^{x} \varphi_{1}\left(x, \rho_{n}\right) \varphi_{1}\left(x, \theta_{n}\right) d t, & x<a, \\ \int_{0}^{a} \varphi_{1}\left(x, \rho_{n}\right) \varphi_{1}\left(x, \theta_{n}\right) d t+\int_{a}^{x} \varphi_{2}\left(x, \rho_{n}\right) \varphi_{2}\left(x, \theta_{n}\right) d t, & x>a,\end{cases}
$$

where $\rho_{n}^{2}=\lambda_{n}$ and $\theta_{n}^{2}=\mu_{n}$.

Lemma 3.2, Let $\rho=\sigma+i \tau$. The following estimates hold

$$
|D(x, \lambda, \mu)|,|\widetilde{D}(x, \lambda, \mu)| \leq \frac{C_{x} \exp (|\tau| x)}{|\rho \pm \theta|+1}, \mu=\theta^{2} \geq 0, \pm \theta \Re \rho \geq 0
$$

Proof, For definiteness, let $\theta \geq 0$ and $\sigma \geq 0$. All other cases can be treated in the same way. Take a fixed $\delta_{0}$. For $|\rho-\theta| \geq \delta_{0}$ we have by virtue of (3.9) and relation of $\left|\varphi^{v}(x, \lambda)\right| \leq C|\rho|^{v} \exp (|\tau| x)$, for $v=0,1$

$$
|D(x, \lambda, \mu)|=\left|\frac{W(\varphi(x, \lambda), \varphi(x, \mu))}{\lambda-\mu}\right| \leq \operatorname{Cexp}(|\tau| x) \frac{|\rho|+|\theta|}{\left|\rho^{2}-\theta^{2}\right|^{.}}
$$

Where $C$ positive constant

Since

$$
\frac{|\rho|+|\theta|}{|\rho+\theta|}=\frac{\sqrt{\sigma^{2}+\tau^{2}}+\theta}{\sqrt{(\sigma+\theta)^{2}+\tau^{2}}} \leq \frac{\sqrt{\sigma^{2}+\tau^{2}}+\theta}{\sqrt{\sigma^{2}+\tau^{2}+\theta^{2}}} \leq \sqrt{2} .
$$


So, we have

$$
|D(x, \lambda, \mu)| \leq \frac{\operatorname{Cexp}(|\tau| x)}{|\tau-\theta|}
$$

For $|\rho-\theta| \geq \delta_{0}$ we get

$$
\frac{|\rho-\theta|+1}{|\rho-\theta|} \leq 1+\frac{1}{\delta_{0}}
$$

And consequently

$$
\frac{1}{|\rho-\theta|} \leq \frac{C_{0}}{|\rho-\theta|+1}, C_{0}=\frac{\delta_{0}+1}{\delta_{0}} .
$$

Substituting this estimate into the right-hand side of (3.13) we obtain

$$
|D(x, \lambda, \mu)| \leq \frac{C \exp (|\tau| x)}{|\rho-\theta|+1}
$$

Therefore,(3.11) is proved for $|\rho-\theta| \geq \delta_{0}$. Analogously, for $|\rho-\theta| \leq \delta_{0}$, (3.11) is valid.

In the following figure we have the contour $\gamma=\gamma^{\prime} \cup \gamma^{\prime \prime}$ where $\gamma^{\prime}$ is a bounded closed contour encircling the set $\left\{\lambda=\rho^{2}: \operatorname{Im} \rho \geq 0, \rho \neq 0: \chi(\rho)=0\right\}$ and $\gamma^{\prime \prime}$ is the two-sided cut along the $\operatorname{arc}\left\{\lambda: \lambda>0, \lambda \notin \operatorname{int} \gamma^{\prime}\right\}$.

Theorem 3.3 The following relations hold

$$
\begin{aligned}
& \tilde{\varphi}(x, \lambda)=\varphi(x, \lambda)+\frac{1}{2 \pi i} \int_{\gamma} \tilde{r}(x, \lambda, \mu) \varphi(x, \mu) d \mu \\
& r(x, \lambda, \mu)-\tilde{r}(x, \lambda, \mu)+\frac{1}{2 \pi i} \int_{\gamma} \tilde{r}(x, \lambda, \xi) r(x, \mu, \xi) d \xi=0 .
\end{aligned}
$$

The relation (3.14) is called the main equation of the inverse problem.

Proof. For $\lambda, \mu \in \gamma, \pm \operatorname{Re} \rho, \operatorname{Re} \theta \geq 0$,

$$
|r(x, \lambda, \mu)|,|\tilde{r}(x, \lambda, \mu)| \leq \frac{C_{x}}{|\mu|(|\rho \pm \theta|+1)},|\varphi(x, \lambda)| \leq C .
$$

Denote $\mathrm{J}_{\gamma}=\left\{\lambda: \lambda \notin \gamma \cup \gamma^{\prime}\right\}$. Consider the Contour $\gamma_{\mathrm{R}}=\gamma \cap\{\lambda:|\lambda| \leq \mathrm{R}\}$ with counter clockwise circuit, and also consider the contour $\gamma_{\mathrm{R}}^{0}=\gamma_{\mathrm{R}} \cup\{\lambda:|\lambda|=\mathrm{R}\}$ with clockwise circuit. By Cauchy's integral formula

$$
P_{1 k}(x, \lambda)-\delta_{1 k}=\frac{1}{2 \pi i} \int_{\gamma_{R}^{0}} \frac{P_{1 k}(x, \mu)-\delta_{1 k}}{\lambda-\mu} d \mu, \lambda \in \text { int } \gamma_{R}^{0}
$$




$$
\frac{P_{j k}(x, \lambda)-P_{j k}(x, \mu)}{\lambda-\mu}=\frac{1}{2 \pi i} \int_{\gamma_{R}^{0}} \frac{P_{j k}(x, \xi)}{(\lambda-\xi)(\xi-\mu)} d \xi, \quad \mu, \lambda \in \operatorname{int} \gamma_{R}^{0}
$$

Using (3.7) we get

$$
\lim _{R \rightarrow \infty} \int_{|\mu|=R} \frac{P_{1 k}(x, \mu)-\delta_{1 k}}{\lambda-\mu} d \mu=0, \lim _{R \rightarrow \infty} \int \frac{P_{j k}(x, \mu)}{(\lambda-\xi)(\xi-\mu)} d \xi=0,
$$

And consequently

$$
\begin{array}{cc}
P_{1 k}(x, \lambda)=\delta_{1 k}+\int_{\gamma} \frac{P_{1 k}(x, \mu)-\delta_{1 k}}{\lambda-\mu} d \mu, & \lambda \in J_{\gamma} \\
\frac{P_{j k}(x, \lambda)-P_{j k}(x, \mu)}{\lambda-\mu}=\frac{1}{2 \pi i} \int_{\gamma} \frac{P_{j k}(x, \xi)}{(\lambda-\xi)(\xi-\mu)} d \xi, & \mu, \lambda \in \text { int } J_{\gamma}
\end{array}
$$

By virtue of (3.6) and (3.17),

$$
\varphi(x, \lambda)=\tilde{\varphi}(x, \lambda)+\frac{1}{2 \pi i} \int_{\gamma} \frac{\widetilde{\varphi}(x, \lambda) P_{11}(x, \mu)+\widetilde{\varphi}^{\prime}(x, \lambda) P_{12}(x, \mu)}{\lambda-\mu} d \mu, \quad \lambda \in J_{\gamma}
$$

Taking (3.4) into account we get

$$
\begin{aligned}
\varphi(x, \lambda)=\tilde{\varphi}(x, \lambda)+\frac{1}{2 \pi i} \int_{\gamma}[ & {\left[\tilde{\varphi}(x, \lambda)\left(\varphi(x, \lambda) \widetilde{\Phi}^{\prime}(x, \mu)-\Phi(x, \mu) \tilde{\varphi}^{\prime}(x, \mu)\right)\right.} \\
+ & \left.\tilde{\varphi}^{\prime}(x, \lambda)(\Phi(x, \lambda) \tilde{\varphi}(x, \mu)-\varphi(x, \mu) \widetilde{\Phi}(x, \mu))\right] \frac{d \mu}{\lambda-\mu} .
\end{aligned}
$$

In view of (3.1), this yields (3.14). According to (3.18) and the proof of Lemma (1.6.3, in [6]) we arrive at

$D(x, \lambda, \mu)-\widetilde{D}(x, \lambda, \mu)=\frac{1}{2 \pi i} \int_{\gamma}\left(\frac{W(\widetilde{\varphi}(x, \lambda), \widetilde{\Phi}(x, \xi)) W(\varphi(x, \xi), \varphi(x, \mu)}{(\lambda-\xi)-(\xi-\mu)}\right)-\frac{1}{2 \pi i} \int_{\gamma}\left(\frac{W(\widetilde{\varphi}(x, \lambda), \widetilde{\varphi}(x, \xi)) W(\Phi(x, \xi), \varphi(x, \mu)}{(\lambda-\xi)-(\xi-\mu)}\right) d \xi$

In view of (3.1) and (3.9) this yields (3.15).

Theorem 3.4 For each fixed $x \in(0, a) \cup(a, \pi)$, the main equation (3.14) has a unique solution $\varphi(x, \lambda) \in C(\gamma)$. Where $C(\gamma)$ is a Banach space contained the continuous bounded functions $\mathrm{z}(\lambda), \lambda \in \gamma$, with the norm $\|\mathrm{z}\|=\sup _{\lambda \in \gamma}|\mathrm{z}(\lambda)|$.

Proof For $0<x<a$, we consider the following linear bounded operators in $C(\gamma)$

$$
\begin{aligned}
& \tilde{A} z(\lambda)=z(\lambda)+\frac{1}{2 \pi i} \int_{\gamma} \tilde{r}(x, \lambda, \mu) z(\mu) d \mu, \\
& A z(\lambda)=z(\lambda)-\frac{1}{2 \pi i} \int_{\gamma} \tilde{r}(x, \lambda, \mu) z(\mu) d \mu,
\end{aligned}
$$

From (3.19) and (3.20) we get

$$
\tilde{A} A(\lambda)=z(\lambda)+\frac{1}{2 \pi i} \int_{\gamma} \tilde{r}(x, \lambda, \mu) z(\mu) d \mu-\frac{1}{2 \pi i} \int_{\gamma} \tilde{r}(x, \lambda, \mu) z(\mu) d \mu
$$




$$
\begin{gathered}
-\frac{1}{2 \pi i} \int_{\gamma} \tilde{r}(x, \lambda, \xi)\left(\frac{1}{2 \pi i} \int_{\gamma} \tilde{r}(x, \lambda, \mu) z(\mu) d \mu\right) d \xi= \\
=z(\lambda)-\frac{1}{2 \pi i} \int_{\gamma}\left(r(x, \lambda, \mu)-\tilde{r}(x, \lambda, \mu)+\frac{1}{2 \pi i} \int_{\gamma} \tilde{r}(x, \lambda, \mu) r(x, \xi, \mu) d \xi\right) z(\mu) d \mu .
\end{gathered}
$$

From (3.15) we have

$$
A \widetilde{A} z(\lambda)=z(\lambda), z(\lambda) \in C(\lambda) .
$$

Also we obtain analogously $A \widetilde{\mathrm{A}} z(\lambda)=z(\lambda)$.Thus,

$$
\tilde{A} A=A \tilde{A}=E
$$

Where $\mathrm{E}$ is the identity operator, Hence the operator $\tilde{A}$ has a bounded inverse operator, and the main equation (3.14) is uniquely solvable for each fixed $0<x<a$. Analogously For $a<x<\pi$ relation (3.14) is uniquely solvable.

Theorem 3.5 The following relations hold

$$
\begin{gathered}
\mathrm{q}(\mathrm{x})=\tilde{\mathrm{q}}(\mathrm{x})+\varepsilon(\mathrm{x}), \\
\mathrm{h}=\tilde{\mathrm{h}}-\varepsilon_{0}(0) . \\
\mathrm{H}=\widetilde{\mathrm{H}}+\varepsilon_{0}(\pi), \mathrm{H} 2=\widetilde{\mathrm{H}}_{2}+\varepsilon_{0}(\pi) \widetilde{\mathrm{H}}_{1}, \mathrm{H}_{1}=\widetilde{\mathrm{H}}_{1}, \\
\alpha_{2}=\tilde{\alpha}_{2}-\left(\frac{\alpha_{1}^{4}-1}{\alpha_{1}}\right) \varepsilon_{0}(a-0),
\end{gathered}
$$

Where

$$
\varepsilon_{0}(\mathrm{x})=\frac{1}{2 \pi \mathrm{i}} \int_{\gamma} \widetilde{\varphi}(\mathrm{x}, \mu) \varphi(\mathrm{x}, \mu) \mathrm{M}(\mu) \mathrm{d} \mu, \quad \varepsilon(\mathrm{x})=-2 \varepsilon_{0}^{\prime}(\mathrm{x})
$$

Proof. By (3.9), (3.14) and (3.25) we get

$$
\begin{aligned}
& \tilde{\varphi}^{\prime}(x, \lambda)-\varepsilon_{0}(x) \tilde{\varphi}(x, \lambda)=\varphi^{\prime}(x, \lambda)+\frac{1}{2 \pi i} \int_{\gamma} \tilde{r}(x, \lambda, \mu) \varphi^{\prime}(x, \mu) d \mu \\
& \tilde{\varphi}^{\prime \prime}(x, \lambda)=\varphi(x, \lambda)+\frac{1}{2 \pi i} \int_{\gamma} \tilde{r}(x, \lambda, \mu) \varphi^{\prime \prime}(x, \mu) d \mu \\
& +\frac{1}{2 \pi i} \int_{\gamma} 2 \tilde{\varphi}(x, \lambda) \tilde{\varphi}(x, \mu) \varphi^{\prime}(x, \mu) M(\mu) d \mu+\frac{1}{2 \pi i} \int_{\gamma} 2(\tilde{\varphi}(x, \lambda) \tilde{\varphi}(x, \mu))^{\prime} \varphi(x, \mu) M(\mu) d \mu
\end{aligned}
$$

In (3.27) we replace the second derivatives by using equation(1.1), and so we replace $\varphi(x, \lambda)$ using (3.14). This yields

$$
\begin{aligned}
\tilde{q}(x) \tilde{\varphi}(x, \lambda)= & q(x) \tilde{\varphi}(x, \lambda)+\frac{1}{2 \pi i} \int_{\gamma} W(\varphi(x, \lambda), \varphi(x, \mu)) M(\mu) \varphi(x, \mu) d \mu \\
& +\frac{1}{2 \pi i} \int_{\gamma} 2 \tilde{\varphi}(x, \lambda) \tilde{\varphi}(x, \mu) M(\mu) \varphi^{\prime}(x, \mu) d \mu \\
& +\frac{1}{2 \pi i} \int_{\gamma}(\tilde{\varphi}(x, \lambda) \tilde{\varphi}(x, \mu))^{\prime} M(\mu) \varphi(x, \mu) d \mu
\end{aligned}
$$


After canceling terms with $\varphi^{\prime}(x, \lambda)$ we arrive at (3.21). Taking $x=0$ and $x=\pi$ in (3.26) and(3.14) we get (3.22) - (3.23), also By virtue of (1.4), it from (3.25) that

$$
\varepsilon_{0}(a+0)=\alpha_{1}^{2} \varepsilon_{0}(a-0) .
$$

And using (1.4), (3.26) and (3.28) we have (3.24).

Thus, we obtain the following algorithm for the solution of the inverse problem.

Algorithm 3.1 Let the function $M(\lambda)$ be given. Then

i) Choose $\tilde{L}$ such that $\tilde{q}(x) \in L_{2}(0, \pi), \widetilde{h}, \widetilde{H}, \widetilde{H}_{1}, \widetilde{H}_{2}, \tilde{\alpha}_{1}$ and $\tilde{\alpha}_{2} \in \mathbb{R}$;

ii) Find $\varphi(x, \lambda)$ by solving equation (3.14);

iii) Construct $q(x)$ and $h, H, H_{1}, H_{2}$ via (3.21) - (3.23);

iv) Construct $\alpha_{2}$ by (3.24).

\section{Reconstruction by spectral data}

Let two sequences of real numbers $\left\{\lambda_{n}\right\}$ and $\left\{\gamma_{n}\right\},\left(n \in \mathbb{Z}_{+}\right)$with the following

Properties be given

$$
\begin{aligned}
& \sqrt{\lambda_{n}}=\rho_{n}=\rho_{n-3}^{0}+\frac{\delta_{n}}{n}+\frac{\xi_{n}}{n} \\
& \gamma_{n}=\left(\rho_{n-3}^{0}\right)^{4}\left(\frac{\pi-a}{2}\left(\left(\alpha^{+}\right)^{2}+\left(\alpha^{-}\right)^{2}\right)+\frac{a}{2}\right)+O\left(n^{3}\right)
\end{aligned}
$$

Where

$$
\xi_{n}=O(1), \delta_{n} \in l_{\infty}, \alpha^{+}=\alpha_{1}+\frac{1}{\alpha_{1}}, \alpha^{-}=\alpha_{1}-\frac{1}{\alpha_{1}}
$$

Now we consider the inverse problem of recovering $L$ from the spectral data $\left\{\lambda_{n}, \gamma_{n}\right\}_{n \geq 0}$. Let us choose a model boundary value problem $\tilde{L}=L\left(\tilde{q}(x), \tilde{h}, \widetilde{H}, \widetilde{H}_{1}, \widetilde{H}_{2}, \tilde{\alpha}_{1}, \tilde{\alpha}_{2}\right)$ with real $\tilde{q}(x) \in$ $L_{2}(0, \pi), \widetilde{h}, \widetilde{H}, \widetilde{H}_{1}, \widetilde{H}_{2}$ and $\tilde{r}:=\widetilde{H} \widetilde{H}_{1}-\widetilde{H}_{2}>0$ such that $\omega=\widetilde{\omega}$ and $\omega=h+H+\frac{1}{2} \int_{0}^{\pi} q(t) d t$. Let

$$
a=\tilde{a} \quad \text { and } \quad \sum_{n=0}^{\infty} \xi_{n}\left|\rho_{n}\right|<\infty
$$

Where $\xi_{n}=\left|\rho_{n}-\tilde{\rho}_{n}\right|+\left|\gamma_{n}-\tilde{\gamma}_{n}\right|$. Denote

$$
\begin{gathered}
\lambda_{n 0}=\lambda_{n}, \lambda_{n 1}=\tilde{\lambda}_{n}, \gamma_{n 0}=\gamma_{n}, \gamma_{n 1}=\tilde{\gamma}_{n}, \varphi_{n i}(x)=\varphi\left(x, \lambda_{n i}\right), \tilde{\varphi}_{n i}(x)=\tilde{\varphi}\left(x, \lambda_{n i}\right) \\
\tilde{Q}_{k j}(x, \lambda)=\frac{W\left(\widetilde{\varphi}(x, \lambda), \widetilde{\varphi}_{k j}(x)\right)}{\gamma_{k j}\left(\lambda-\lambda_{k j}\right)}=\frac{1}{\gamma_{k j}} \int_{0}^{x} \tilde{\varphi}(t, \lambda), \tilde{\varphi}_{k j}(t) d t \\
\tilde{Q}_{n i, k j}(x, \lambda)=\tilde{Q}_{k j}\left(x, \lambda_{n i}\right)
\end{gathered}
$$

It follows from (2.15),(2.16) that

$$
\left|\varphi_{n j}^{v}(x)\right| \leq C\left(\left|\rho_{n}^{0}+1\right|\right)^{v},\left|\tilde{\varphi}_{n j}^{v}(x)\right| \leq C\left(\left|\rho_{n}^{0}+1\right|\right)^{v}
$$




$$
\left|\tilde{Q}_{n i, k j}(x)\right| \leq \frac{C}{\left|\rho_{n}^{0}-\rho_{k}^{0}\right|+1},\left|\tilde{Q}_{n i, k j}^{v+1}(x)\right| \leq C\left(\left|\rho_{n}^{0}\right|+\left|\rho_{k}^{0}\right|+1\right)^{v} .
$$

Lemma 4.1 The following relation holds

$$
\tilde{\varphi}(x, \lambda)=\varphi(x, \lambda)+\sum_{n=0}^{\infty}\left(\tilde{Q}_{k 0}(x, \lambda) \varphi_{k 0}(x)-\tilde{Q}_{k 1}(x, \lambda) \varphi_{k 1}(x)\right)
$$

Proof By virtue of (4.1) we have

$$
a=\tilde{a}, \alpha_{1}=\tilde{\alpha}_{1}
$$

It follow from (2.15) and (2.16) that

$$
\left|\varphi^{v}(x, \lambda)-\tilde{\varphi}^{v}(x, \lambda)\right| \leq C|\rho|^{v-1} \exp (|\tau| x) .
$$

Similary,

$$
\left|\psi^{v}(x, \lambda)-\tilde{\psi}^{v}(x, \lambda)\right| \leq C|\rho|^{v-1} \exp (|\tau|(T-x)) .
$$

Denote $G_{\delta}^{0}=G_{\delta} \cap \tilde{G}_{\delta}$. From (3.1) and (4.7) we have

$$
\left|\Phi^{v}(x, \lambda)-\widetilde{\Phi}^{v}(x, \lambda)\right| \leq C_{\delta}|\rho|^{v-2} \exp (-|\tau| x), \quad \rho \in G_{\delta}^{0} v=0,1 .
$$

Let $P(x, \lambda)$ be the matrix defined in Theorem 3.1 and $\Gamma=\left\{\lambda=u+i v: u=(2 h)^{-2} v^{2}-h^{2}\right\}$. be the image of the set $\operatorname{Im} \rho= \pm h$ under the mapping $\lambda=\rho^{2}$.Denote $\Gamma_{n}=\Gamma \cap\left\{\lambda:|\lambda| \leq r_{n}\right\}$, and $\Gamma_{n 0}=\Gamma_{n} \cup\left\{\lambda:|\lambda|=r_{n}, \lambda \notin\right.$ int $\left.\Gamma\right\}, \Gamma_{n 1}=\Gamma_{n} \cup\left\{\lambda:|\lambda|=r_{n}, \lambda \in\right.$ int $\left.\Gamma\right\}$. Since for each fixed $x$, the functions $P_{1 k}$ are meromorphic in $\lambda$ with simple poles $\lambda_{n}$ and $\tilde{\lambda}_{n}$, we get by Cauchy theorem

$$
P_{1 k}(x, \lambda)-\delta_{1 k}=\frac{1}{2 \pi i} \int_{\Gamma_{n 0}} \frac{P_{1 k}(x, \xi)-\delta_{1 k}}{\xi-\lambda} d \xi, \quad k=1,2 .
$$

Where $\lambda \in \Gamma_{n 0}$, and $\delta_{j k}$ is the Kronecker's delta. Further, (3.2) and (3.5) imply

$$
\text { (4.10) } P_{11}(x, \lambda)=1+(\varphi(x, \lambda)-\tilde{\varphi}(x, \lambda)) \widetilde{\Phi}^{\prime}(x, \lambda)-(\Phi(x, \lambda)-\widetilde{\Phi}(x, \lambda)) \widetilde{\varphi}^{\prime}(x, \lambda)
$$

Also we can obtain

$$
\left|P_{1 k}(x, \lambda)-\delta_{1 k}\right| \leq C_{\delta}|\rho|^{-1}, \rho \in G_{\delta}^{0} .
$$

By virtue of (4.11)

$$
\lim _{n \rightarrow \infty} \frac{1}{2 \pi i} \int_{|\xi|=r_{n}} \frac{P_{1 k}(x, \xi)-\delta_{1 k}}{\xi-\lambda} d \xi=0,
$$

And consequently, (4.9) yields

$$
P_{1 k}(x, \lambda)-\delta_{1 k}=\lim _{n \rightarrow \infty} \frac{1}{2 \pi i} \int_{\Gamma_{n 1}} \frac{P_{1 k}(x, \xi)-\delta_{1 k}}{\xi-\lambda} d \xi .
$$

Substituting into (3.6) we obtain

$$
\varphi(x, \lambda)=\tilde{\varphi}(x, \lambda)+\lim _{n \rightarrow \infty} \frac{1}{2 \pi i} \int_{\Gamma_{n 1}} \frac{\tilde{\varphi}(x, \lambda) P_{11}(x, \xi)+\tilde{\varphi}^{\prime}(x, \lambda) P_{12}(x, \xi)}{\xi-\lambda} d \xi .
$$

Taking (3.5) into account we calculate

$$
\varphi(x, \lambda)=\tilde{\varphi}(x, \lambda)+\lim _{n \rightarrow \infty} \frac{1}{2 \pi i} \int_{\Gamma_{n 1}}\left(\tilde{\varphi}(x, \lambda)\left(\varphi(x, \xi) \widetilde{\Phi}^{\prime}(x, \xi)-\Phi(x, \xi) \tilde{\varphi}^{\prime}(x, \xi)\right)\right.
$$




$$
+\tilde{\varphi}^{\prime}(x, \lambda)(\Phi(x, \xi) \tilde{\varphi}(x, \xi)-\varphi(x, \xi) \widetilde{\Phi}(x, \xi)) \frac{d \xi}{\lambda-\xi^{\prime}}
$$

Or, in view of (3.1),

$$
\tilde{\varphi}(x, \lambda)=\varphi(x, \lambda)+\lim _{n \rightarrow \infty} \frac{1}{2 \pi i} \int_{\Gamma_{n 1}} \frac{W(\widetilde{\varphi}(x, \lambda), \widetilde{\varphi}(x, \xi))}{\lambda-\xi} M(\xi) \varphi(x, \xi) d \xi .
$$

Then we have

$$
\operatorname{Res}_{\xi=\lambda_{k j}} \frac{W(\widetilde{\varphi}(x, \lambda), \widetilde{\varphi}(x, \xi))}{\lambda-\xi} M(\lambda) \varphi(x, \xi)=\tilde{Q}_{k j}(x, \lambda) \varphi_{k j}(x)
$$

Now with calculation the integral in (4.13) by residue theorem we arrive at (4.4).

Let $K$ be a set of indices $n=(n, i), n \geq 0, i=0,1$. For each fixed $x \in[0, \pi]$, we define the vector

$$
\psi(x)=\left[\psi_{u}(x)\right]_{u \in K}=\left[\begin{array}{l}
\psi_{n 0}(x) \\
\psi_{n 1}(x)
\end{array}\right]_{n \geq 0}=\left[\psi_{00}, \psi_{01}, \psi_{02, \ldots}\right]^{T}
$$

by the formulae

$$
\begin{gathered}
{\left[\begin{array}{l}
\psi_{n 0}(x) \\
\psi_{n 1}(x)
\end{array}\right]_{n \geq 0}=\left[\begin{array}{cc}
\chi_{n} & -\chi_{n} \\
0 & 1
\end{array}\right]\left[\begin{array}{l}
\varphi_{n 0}(x) \\
\varphi_{n 1}(x)
\end{array}\right]=\left[\begin{array}{c}
\chi_{n}\left(\varphi_{n 0}(x)-\varphi_{n 1}(x)\right) \\
\varphi_{n 1}(x)
\end{array}\right],} \\
\chi_{n}= \begin{cases}\xi_{n}^{-1} & \xi_{n} \neq 0 \\
0 & \xi_{n}=0\end{cases}
\end{gathered}
$$

we also define the block matrix

$$
H(x)=\left[H_{u, v}(x)\right]_{u, v \in K}=\left[\begin{array}{ll}
H_{n 0, k 0}(x) & H_{n 0, k 1}(x) \\
H_{n 1, k 0}(x) & H_{n 1, k 1}(x)
\end{array}\right] \cdot n, k \geq 0, u=(n, i), v=(k, j)
$$

By the formulae

$$
\begin{aligned}
& {\left[\begin{array}{cc}
H_{n 0, k 0}(x) & H_{n 0, k 1}(x) \\
H_{n 1, k 0}(x) & H_{n 1, k 1}(x)
\end{array}\right]=\left[\begin{array}{cc}
\chi_{n} & -\chi_{n} \\
0 & 1
\end{array}\right]\left[\begin{array}{ll}
Q_{n 0, k 0}(x) & Q_{n 0, k 1}(x) \\
Q_{n 1, k 0}(x) & Q_{n 1, k 1}(x)
\end{array}\right]\left[\begin{array}{cc}
\xi_{k} & 1 \\
0 & -1
\end{array}\right]} \\
& =\left[\begin{array}{cc}
\xi_{n} \chi_{n}\left(Q_{n 0, k 0}(x)-Q_{n 1, k 0}(x)\right) & \chi_{n}\left(Q_{n 0, k 0}(x)-Q_{n 0, k 1}(x)-Q_{n 1, k 0}(x)+Q_{n 1, k 1}(x)\right) \\
\xi_{k} Q_{n 1, k 0}(x) & Q_{n 1, k 0}(x)-Q_{n 1, k 1}(x)
\end{array}\right]
\end{aligned}
$$

Analogously we define $\tilde{\psi}(x), \widetilde{H}(x)$ by replacing in the previous definitions, $\varphi_{n i}(x)$ by $\tilde{\varphi}_{n i}(x)$ and $Q_{n i, k j}(x)$ by $\tilde{Q}_{n i, k j}(x)$ also we have

$$
\left|\psi_{n i}^{v}(x)\right| \leq C\left(\rho_{n}^{0}+1\right)^{v},\left|H_{n i, k j}(x)\right| \leq \frac{C \xi_{k}}{\left|\rho_{n}^{0}-\rho_{k}^{0}\right|+1}
$$

Similary

$$
\left|\tilde{\psi}_{n i}^{v}(x)\right| \leq C\left(\rho_{n}^{0}+1\right)^{v},\left|\widetilde{H}_{n i, k j}(x)\right| \leq \frac{C \xi_{k}}{\left|\rho_{n}^{0}-\rho_{k}^{0}\right|+1}
$$


Let us consider the Banach space $m$ of bounded squencess $\alpha=\left[\alpha_{u}\right]_{u \in K}$ with the norm $\|\alpha\|_{m}=$ $\sup _{u \in K}\left|\alpha_{\mathrm{u}}\right|$. It follows from (4.14) and (4.15) that for each fixed $x \in[0, \pi]$, the operators $E+\widetilde{H}(x)$ and $E-\widetilde{H}(x)$ (here $E$ is the identity operator), acting from $m$ to $m$, is a linear bounded operator, and

$$
\|\widetilde{H}(x)\| \leq \sup _{n} \sum_{k} \frac{\xi_{k}}{\left|\rho_{n}^{0}-\rho_{k}^{0}\right|+1}<\infty
$$

Taking into account our notation, we can rewrite (4.4) in the form

$$
\tilde{\psi}_{n i}(x, \lambda)=\psi_{n i}(x, \lambda)+\sum_{n=0}^{\infty}\left(\widetilde{H}_{n i, k 0}(x) \psi_{k 0}(x)-\widetilde{H}_{n i, k 1}(x) \psi_{k 1}(x)\right)
$$

Or

$$
\tilde{\psi}(x, \lambda)=(E+\widetilde{H}(x)) \psi(x)
$$

Thus, for each fixed $x$, the vector $\psi(x) \in m$ is a solution of equation (4.16)in the Banach space $m$. Equation (4.16) is called the main equation of the inverse problem. solving (4.16) we find the vector $\psi(x)$ and consequently, the functions $\varphi_{n i}(x), n \geq 0, i=0,1$. Since $\varphi_{n i}(x)=\varphi\left(x, \lambda_{n i}\right)$ are the solutions of (1.1), we can construct the function $q(x)$ by the formula

$$
q(x)=\lambda_{n i}+\frac{\varphi_{n i}^{\prime \prime}(x)}{\varphi_{n i}(x)} .
$$

we get the coefficient $h$ by

$$
h=\varphi^{\prime}\left(0, \lambda_{n i}\right) .
$$

Also we obtain the coefficients $H, H_{1}$ and $H_{2}$ from the linear system of equations

$$
\left\{\begin{array}{l}
\left(\lambda_{n 0}-H_{1}\right) \varphi_{n 0}^{\prime}(\pi)+\left(\lambda_{n 0} H-H_{2}\right) \varphi_{n 0}(\pi)=0 \quad n \geq 0, \\
\widetilde{\omega}=\omega
\end{array}\right.
$$

And finally we obtain

$$
\alpha_{2}=\frac{\varphi_{n i}^{\prime}(a+0)}{\varphi_{n i}(a-0)}-\frac{\varphi_{n i}^{\prime}(a-0)}{\varphi_{n i}(a+0)}
$$

Now, we get the following algorithm for the solution of the inverse problem of recovering $L$ from the given spectral data $\left\{\lambda_{n}, \gamma_{n}\right\}_{n \geq 0}$.

Algorithm 4.1 Let the spectral data $\left\{\lambda_{n}, \gamma_{n}\right\}_{n \geq 0}$ be given. Then

i) Choose $\tilde{L}$ such that $\widetilde{\omega}=\omega$, and construct $\widetilde{\psi}(x)$ and $\widetilde{H}(x)$;

ii) Find $\psi(x)$ by solving (4.16);

iii) Calculate $q(x), h, H, H_{1}$, and $H_{2}$ by (4.17), (4.18) and (4.19);

iv) Construct $\alpha_{2}$ by (4.20). 
Example 4.2 Take $\tilde{L}=L\left(\tilde{q}(x)=0, a, \alpha_{1}, 0,0,0, \widetilde{H}_{2}<0\right)$. Let $\left\{\lambda_{n}, \gamma_{n}\right\}_{n \geq 0}$. be the spectral data of $\tilde{L}$. Clearly,

$$
\tilde{\lambda}_{0}=0, \tilde{\gamma}_{0}=a+(\pi-a) \alpha_{1}, \tilde{\varphi}_{00}(x)=1(x<a), \tilde{\varphi}_{00}(x)=\alpha_{1}(x>a) .
$$

Let $\lambda_{n}=\tilde{\lambda}_{n}(n \geq 0), \gamma_{n}=\tilde{\gamma}_{n}$, and $\gamma_{0}>0$ be an arbitrary positive number. Denote

$A:=\frac{1}{\gamma_{0}}-\frac{1}{\widetilde{\gamma}_{0}}$, then (4.4) yields

$$
\tilde{\varphi}_{00}(x)=\varphi_{00}(x)\left(1+A \int_{0}^{x} \tilde{\varphi}_{00}^{2}(t) d t\right)
$$

So, we have

$$
\varphi_{00}(x)= \begin{cases}(1+A x),{ }^{-1} & x<a \\ \alpha_{1}\left(B+A \alpha_{1}^{2} x\right),-1 & x>a\end{cases}
$$

Where $B=1+A a-A \alpha_{1}^{2} a$. Using (4.17) and value $\lambda_{00}=0$, it is easy to see that

$$
q(x)=\left\{\begin{array}{lr}
\left.2 A^{2}(1+A x)\right)^{-2} & x<a \\
2 A^{2} \alpha_{1}^{4}\left(B+A \alpha_{1}^{2} x\right),^{-2} & x>a
\end{array}\right.
$$

Also, we can obtain the following relations

$$
h=-A, \quad H=\frac{A \alpha_{1}^{2}}{\left(B+A \alpha_{1}^{2} \pi\right)}, \quad H_{1}=\frac{\alpha_{1}}{\left(B+A \alpha_{1}^{2} \pi\right)} \quad H_{2}=\frac{-A \alpha_{1}^{3}}{\left(B+A \alpha_{1}^{2} \pi\right)^{2}}
$$

So finally we obtain

$$
\alpha_{2}=\frac{\varphi_{n i}^{\prime}(a+0)}{\varphi_{n i}(a-0)}-\frac{\varphi_{n i}^{\prime}(a-0)}{\varphi_{n i}(a+0)}=\frac{A\left(\alpha_{1}^{-1}-\alpha_{1}^{3}\right)}{(1+A a)}
$$

\section{References}

[1] G. Freiling and V. Yurko, Inverse problem for Sturm-Liouville problems and their Applications, Nova Science, New York, 2001.

[2] N. J. Guliyev, Inverse eigenvalue problems for Sturm-Liouville equations with spectral parameter linearly contained in one of the boundary conditions, Inverse problems, 21(2005), 1315-1330.

[3] J. Walter, Regular eigenvalue problems with eigenvalue parameter in the boundary condition, Math Zeitschrift, 133(1973), 301-312.

[4] C. T. Fulton, Two-point boundary value problems with eigenvalue parameter contained in the boundary conditions, Proc. R. Soc. Edinburg, A77 (1977), 293-308.

[5] C. T. Fulton, Singular eigenvalue problems with eigenvalue parameter contained in the boundary conditions, proc. R. Soc. Edinburg, B87 (1980),1-34

[6] B. Keskin, A. Sinan and N. Yalkin, Inverse spectral problems for discontinuous Sturm-Liouville operator with eigenvalue dependent boundary conditions, volume 60, Number 1(2011), 15-25.

[7] B. M. Levitan and I. S. Sargsyan, Introduction to spectral Theory, American Mathematical Society, Providence, RI, USA, 1975.

[8] D. G. Shepelsky, The inverse problem of reconstruction of the medium conductivity in a class of discontinuous and increasing functions, Spectral operator theory and related topics, 209-232, Advances in Soviet Math., 19, Amer. Math. Soc., providence, RI.1994 
[9] E. M. Russakovsk, Operator treatment of boundary problems with spectral parameters entering via polynomials in the boundary conditoins, Funct. Anal. Appl., 9(1975), 358-359.

[10] E. A. Codington and N. Levinson, Theory of Ordinary Differential Equations, McGraw-Hill, New York, NY, USA, 1955.

[11] R. kh. Amirov, On Sturm-Liouville operators with discontinuity conditions inside an interval, J. Math. Anal. Appl., 317(2006), 163-176.

[12] I. M. Gelfand and B. M. Levitan, On the determination of a differential equation from its spectral function Amer, Math. Soc. Transl. Ser. 21(1955), 253-304.

[13] Mohammad Shahriari, Aliasghar Jodayree Akbarfama, and Gerald Teschl, Uniqueness for Inverse Sturm-Liouville Problems with a Finite Number of Transmission Conditions, J.Math. Anal. Appl., 395 (2012), 19-29.

[14] N. B. kerimov and V. S. Mirzoev, On the basis properties of one spectral parameter in the boundary conditions, 33 (1997), 116-120,

[15] P. J. Browne and B. D. Sleeman, A uniqueness theorem for inverse eigenparameter dependent Sturm-Liouville problems, Inverse Problems, 13(6)(1997), 1453-1462.

[16] V. A. Yurko, Reconstruction of singular non-self-adjoint differential operators with a singularity inside an interval, Differential Equations, 38(5) (2002) 678-694.

[17] V. A. Yurko, Inverse Spectral problems for Linear Differential Operators and their Applications, Gordon and Breach, New York, 2000

[18] O. H. Hald, Discontinuous inverse eigenvalue problems, Comm. Pure Appl. Math., 37(1984), 539577.

[19] Marchenko V A 1977, The Sturm-Liouville operators and their applications(Russian), (Kiev:Naukova Dumka) English transl. :(Basel: Birkhauser Verlag, 1986)

[20] R. S. Anderssen, The effect of discontinuities in density and shear velocity on the asymptotic overtone structure of to rational eigenfrequencies of the earth, Geophys. J. R. astr. Soc., 50 (1997), 303309.

[21] G. Teschl, Mathematical Methods in Quantum Mechanics; With Applications to Schrödinger Operators, Graduate Studies in Mathematics, Amer. Math. Soc., Rhode Island, 2009. 